\title{
AN AUDIT OF MANAGEMENT OF RESPIRATORY DISTRESS SYNDROME IN THE CONTEXT OF THE 2007 EUROPEAN CONSENSUS GUIDELINES
}

\author{
A. Ahmad Kamar ${ }^{1,2}$, L. Machen ${ }^{2}$, D.M. Sheridan-Pereira ${ }^{2}$ \\ ${ }^{1}$ Rotunda Hospital, ${ }^{2}$ Coombe Women and Infants University Hospital, Dublin, Ireland
}

Background and aims: To audit compliance and variation in management of neonatal respiratory distress syndrome (RDS) in an era post publication of the 2007 European Consensus Guidelines.

Methodology: Retrospective review of inborn preterms less than $(<) 37$ weeks with RDS for the period from January $1^{\text {st }} 2008$ to December $31^{\text {st }} 2008$.

Results: Of 197 infants, 29(14.72\%) infants were $<27$ weeks, 31(15.74\%) between 27 to $<30$ weeks, $91(46.19 \%)$ between 30 to $<34$ weeks, and $46(23.35 \%)$ between 34 to $<37$ weeks. All infants $<27$ weeks received surfactant, administered before $15 \mathrm{mins}$ in 28 of 29 infants. $80.65 \%$ infants in the 27 to $<30$ weeks age group received surfactant, in comparison to $37.36 \%$ of infants between 30 to $<34$ weeks, and $15.22 \%$ infants between 34 to $<37$ weeks. Prophylactic surfactant was administered in $58 \%$ of infants between 27 to $<30$ weeks age group. This did not correlate with the use or non-use of prenatal steroids. Only 1 of 29 infants $<27$ weeks was immediately extubated post-surfactant. Timing of surfactant re-dosing was variable and initiated at a higher mean $\mathrm{FiO}_{2} 66 \%$ and alveolar-arterial ratio 0.14 in prohylaxed infants $<27$ weeks compared to rescue/re-dosing surfactant at mean $\mathrm{FiO}_{2} 48 \%$ in infants between 27 to $<30$ weeks.

Conclusions: Overall compliance was achieved in prophylactic surfactant administration in infants $<27$ weeks. For babies more than 27 weeks, significant variation was demonstrated in management strategies. This practice variation prompts generation of a unit policy of when to intervene with progression of RDS. 